\title{
Development of ionic-complex-based nanostructured lipid carriers to improve the pharmacokinetic profiles of breviscapine
}

\author{
Mei $\mathrm{LI}^{1}$, Yong ZHENG ${ }^{2}$, Feng-ying SHAN ${ }^{1}$, Jing ZHOU ${ }^{1}$, Tao GONG ${ }^{1, *}$, Zhi-rong ZHANG ${ }^{1}$ \\ ${ }^{1}$ Key Laboratory of Drug Targeting and Drug Delivery Systems, Ministry of Education, West China School of Pharmacy, Sichuan Univer- \\ sity, Chengdu 610041, China; ${ }^{2}$ The Second Affiliated Hospital of Chongqing Medical University, Chongqing 404100, China
}

\begin{abstract}
Aim: Breviscapine isolated from the Chinese herb Erigeron breviscapus (Vant) Hand-Mazz is widely used to treat cardiovascular and cerebrovascular diseases. The aim of this study was to improve the pharmacokinetic profiles of breviscapine using nanostructured lipid carrier based on an ionic complex formation.

Methods: Breviscapine nanostructured lipid carrier (Bre-NLC) was prepared using the thin film homogenization method. The morphology of Bre-NLCs was determined using transmission electron microscopy. The mean particle size, polydispersity index, zeta-potential analysis and entrapment efficiency were analized. In vitro release was studied using the dialysis method. In vitro stability was studied in fresh plasma and liver slurry of rats. In vivo pharmacokinetics was analyzed in rats after intravenous injection of a dose equivalent to breviscapine $(10 \mathrm{mg} / \mathrm{kg})$.

Results: The Bre-NLCs were spherical with a mean particle size of $\sim 170 \mathrm{~nm}$, a zeta potential of $\sim 20 \mathrm{mV}$ and a high entrapment efficiency of $\sim 89 \%$. Compared with a commercially available solution, a substantial decrease in the cumulative release of breviscapine was found for the Bre-NLCs. The NLC has a significantly protective effect against the liver enzyme degradation of breviscapine. After intravenous administration in rats, the Bre-NLCs exhibited a 32 times increase in the $\mathrm{AUC}_{0-\mathrm{t}}$ and a 12 times increase in $T_{1 / 2}$ as compared to the commercially available breviscapine solution.

Conclusion: The results demonstrate that the NLC has great potential to use as a novel sustained release system for breviscapine.
\end{abstract}

Keywords: breviscapine; octadecylamine; ionic complex; nanostructured lipid carriers; sustained release; pharmacokinetics

Acta Pharmacologica Sinica (2013) 34: 1108-1115; doi: 10.1038/aps.2013.43; published online 17 Jun 2013

\section{Introduction}

Breviscapine, which is a type of flavone drug, can be isolated from the Chinese herb Erigeron breviscapus (Vant) Hand-Mazz. The main active ingredient in breviscapine is scutellarin-7$o$-glucuronide (Figure 1$)^{[1]}$. It has been reported that breviscapine can dilate blood vessels, improve microcirculation, regulate lipid metabolism, reduce platelet aggregation and scavenge oxygen free radicals. Breviscapine has been widely used to treat cardiovascular and cerebrovascular diseases and to protect the kidney and the liver ${ }^{[2,3]}$. For these chronic diseases, long term treatment is needed. The oral bioavailability of breviscapine is extremely low, $0.40 \% \pm 0.19 \%$ in beagle dogs and $0.18 \% \pm 0.15 \%$ in rats. Intravenous injection is the most commonly used formulation in clinical treatments. However, the fast metabolize rate, short elimination half-life, short blood

\footnotetext{
* To whom correspondence should be addressed.

E-mail gongtaoy@126.com

Received 2013-02-02 Accepted 2013-03-27
}

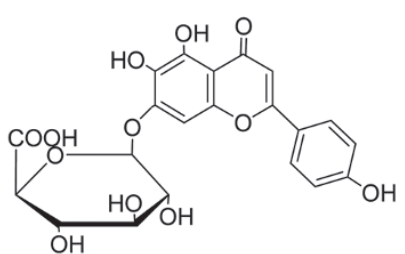

Figure 1. The main active ingredient of breviscapine (2S,3R,4R,5R)6\{[5,6-dihydroxy-2-(4-hydroxyphenyl)-4-oxo-4H-chromen-7-yl]methoxy\}3,4,5-trihydroxy-tetrahydro-2H-pyran-2-carboxylic acid.

residence time and low AUC limit the wide application of breviscapine $^{[4]}$.

Delivery systems have been developed to increase the blood drug concentration and to prolong the circulation time of breviscapine, including multivesicular liposomes ${ }^{[5]}$, unilamellar vesicles $^{[6]}$ and polymeric nanoparticles ${ }^{[7]}$. Despite their great success, there are some limitations to these systems. The lipo- 
some components are biocompatible, but the manufacturing process is complex and has a high $\cos ^{[8]}$. The safety of polymers is a big concern, and there are very few polymers that have been approved for injection in clinics ${ }^{[9,10]}$. Multivesicular liposomes (MVLs), which have a complicated manufacturing process, can only be used for parenteral administration rather than intravenous ${ }^{[11,12]}$. Most importantly, even though there was an increase in the AUC for these systems, there was no significant prolongation of the circulation time. A satisfactory system that could exhibit a good pharmacokinetic profile has not been found in the field of colloidal particle delivery systems for breviscapine. Therefore, a breviscapine delivery system with low toxicity, an easy manufacturing process and an improved pharmacokinetic profile is needed.

Nanostructured lipid carriers (NLC) that are developed from solid lipid nanoparticles (SLN) are mainly composed of biocompatible solid lipid matrices (phospholipids) and liquid lipids (oils). These carriers are natural products with good biocompatibility, low toxicity and low cost. Additionally, the manufacturing of NLC can easily be scaled up from the lab to large industry ${ }^{[13]}$. Most importantly, NLC can entrap lipophilic drugs in the lipid phase, thus providing a slow release of the drug. Insoluble drug-lipid conjugate bulks have been prepared for the incorporation of drugs ${ }^{[14,15]}$. Additionally, the lipids around the drugs might protect the drugs from oxidation, hydrolysis and enzymatic degradation. Generally, NLC can increase the drug stability and provide a sustained release, which might prolong the drug circulation time and improve the therapeutic efficacy.

It is a challenge to encapsulate a poor lipophilic drug in NLC because of the poor compatibility of the matrices. To increase the lipophilicity of breviscapine, octadecylamine was selected as a complexing agent to prepare an ionic complex. Ionic complexes have been widely studied for many years ${ }^{[16-18]}$. This complex would change some of the drug properties, including the hydrophilicity/lipophilicity and the drug release rate ${ }^{[19,20]}$. Ionic complexes can be generated by a variety of chemical bonds, such as hydrogen bonding, electrostatic interactions ${ }^{[21]}$ and van der Waals bonding ${ }^{[22]}$. There is an amine group in octadecylamine and a carboxylic acid group in breviscapine. These two groups can form zwitterionic structures. The lipophilicity of breviscapine could thus be increased, facilitating incorporation into NLC.

The objective of this study is to develop a novel breviscapine-nanostructured lipid carrier (Bre-NLC) to improve the pharmacokinetic profiles of breviscapine, including a prolonged circulation time and an increased AUC. To the best of our knowledge, a long circulating Bre-NLC has not yet been reported. It is anticipated that this study can offer not only a new method for preparing a breviscapine-loaded NLC with a high drug entrapment efficiency but also offer sustained release in vivo.

\section{Materials and methods} Materials

Breviscapine (which contained scutellarin 95\%-105\%) was provided by Jiexiang Herb Co, Ltd (Chengdu, China). Egg yolk lecithin 80 (E80) was supplied by Lipoid Co, Ltd (Ludwigshafen, Germany). Medium-chain fatty acid esters (MCT) were purchased from Tieling Beiya Pharmaceutical Oil Co, Ltd (Tieling, China). Octadecylamine (ODA) was offered by Shengke (Shengke Chemical Co, Ltd, Jining, China). All other chemicals were purchased from Kermel Co, Ltd (Tianjin, China). Ethanol and dichloromethane were analytical grade. Methanol and acetonitrile were HPLC grade. Male Wistar rats $(200 \pm 20 \mathrm{~g})$ were purchased from the Experimental Animal Center of Sichuan University for a pharmacokinetic study.

\section{Analysis method}

The concentration of breviscapine was determined for an in vitro study by a UV detector (Waters, USA). The analysis of breviscapine in plasma was performed using a Shimadzu HPLC system (Kyoto, Japan) and the UV detector equipped with a C18 analytical column $(150 \mathrm{~mm} \times 4.6 \mathrm{~mm}, 5 \mu \mathrm{m})$. The mobile phase consisted of $0.2 \%$ phosphoric acid, acetonitrile and methanol $(65 / 10 / 25, v / v / v)$ at a flow rate of $1.0 \mathrm{~mL} / \mathrm{min}^{[23]}$. A processed plasma sample $(20 \mu \mathrm{L})$ was injected into the analysis system. The effluents were monitored at $335 \mathrm{~nm}$ under $35^{\circ} \mathrm{C}$ and were quantified by a standard curve.

\section{Preparation of NLC}

A Bre-NLC was prepared using a three-step method: the preparation of a phospholipid complex and the formation of an ionic complex and of nanostructured lipid carriers. The procedures are described below.

First, a phospholipid complex was prepared to increase the compatibility of breviscapine with octadecylamine. Breviscapine $(2-5 \mathrm{mg} / \mathrm{mL})$ and lecithin were placed into $100 \%$ ethanol with a mass ratio of 1:3. The system was kept under constant stirring for approximately $1.5 \mathrm{~h}$ at $50^{\circ} \mathrm{C}$. The ethanol was then removed by rotary evaporation (Büchi Rotavapor R-3; Büchi, Switzerland).

Second, all of the other excipients, including MCT, ODA and the remaining phospholipid, were added to the phospholipid complex with $10 \mathrm{~mL}$ of dichloromethane as a solvent. The mixture was stirred at room temperature until it was completely dissolved. During this process, an ionic complex formed. The negatively charged carboxylic acid group in breviscapine and the positively charged ammonium group in ODA effectively formed an ionic complex in a gentle environment. Within the organic solvent, the ionic complexes were incorporated into a lipid matrix. Afterwards, dichloromethane was removed at room temperature, and the ionic complex was obtained.

Third, nanoparticles were prepared using the thin film homogenization method ${ }^{[24]}$. Water, $10 \mathrm{~mL}$, was added to dissolve the membrane. The mixture was then high-pressure homogenized to obtain uniform NLC.

\section{Formulation optimization}

The NLC formulation was optimized by an orthogonal design, which is widely used for formulation screening and optimiza- 
tion $^{[25,26]}$. The researched factors included breviscapine, E80, MCT and ODA. Nine combinations with different concentrations were prepared according to the orthogonal design (Table 1). The factors with fixed ratios were made into NLC using the method described above. The polydispersity index (PDI) and the average size of each sample were determined by a Malvern Nano-ZS90 laser particle size analyzer (Nano-ZS, Malvern Instruments Co, Ltd, Malvern, Worcestershire, UK). All measurements were performed in triplicate.

Table 1. Factors and levels of $\mathrm{L} 9\left(3^{4}\right)$ orthogonal test.

\begin{tabular}{lcccc}
\hline Run & Bre $(\mathrm{mg})$ & E80 $(\mathrm{mg})$ & MCT $(\mathrm{mg})$ & ODA $(\mathrm{mg})$ \\
\hline 1 & 8 & 100 & 20 & 4 \\
2 & 8 & 120 & 40 & 6 \\
3 & 8 & 140 & 60 & 8 \\
4 & 10 & 100 & 40 & 8 \\
5 & 10 & 120 & 60 & 4 \\
6 & 10 & 140 & 20 & 6 \\
7 & 12 & 100 & 60 & 6 \\
8 & 12 & 120 & 20 & 8 \\
9 & 12 & 140 & 40 & 4 \\
\hline
\end{tabular}

Bre, breviscapine; E80, egg yolk lecithin 80; MCT, medium-chain fatty acid esters; ODA, octadecylamine

\section{Encapsulation efficiency}

Ultrafiltration was used to measure the entrapment efficiency ${ }^{[27]}$. Briefly, $0.6 \mathrm{~mL}$ of an undiluted sample was placed in the inner chamber of a centrifuge tube that was matched with an ultrafilter (molecular weight cutoff of $300 \mathrm{kDa}$ ). The nanosuspension was then centrifuged at $3300 \times g$ for $90 \mathrm{~min}$ at $4{ }^{\circ} \mathrm{C}$. The encapsulated breviscapine remained in the inner chamber, whereas the unloaded breviscapine moved to the outer chamber through the filter membrane. The drug content was determined using an UV assay. The average entrapment efficiency was calculated from three measurements using the following equation:

$$
\mathrm{EE} \%=(1-\text { free drug } / \text { total drug }) \times 100 \%
$$

\section{Particle size}

The particle size and polydispersity index (PDI) were measured by the Malvern Nano-ZS90 laser particle size analyzer at $25^{\circ} \mathrm{C}$. Prior to size analysis, the sample was diluted with distilled water 20-fold. The index of refraction was 1.330. For the zeta potential $(\zeta)$ analysis, the sample was used in its original concentration, ie, without dilution.

\section{Transmission electron microscopy (TEM)}

The morphology of the Bre-NLC was determined by TEM. The nanoparticle suspension was diluted three times with distilled water. The diluted suspension, $20 \mu \mathrm{L}$, was dropped onto carbon-coated polyester-fiber-supported copper grids. The absorption process lasted for approximately $10 \mathrm{~min}$. The nanoparticles were then negatively stained with $1 \%$ sodium phosphotungstate for $5 \mathrm{~min}$. After the excess solution was removed by a filter paper, the copper grid was placed in the TEM for examination.

\section{In vitro release study}

An in vitro release study was completed using the dialysis method. A Bre-NLC suspension or a commercial drug solution, $1 \mathrm{~mL}$, was sealed in a dialysis bag (8000-14000 Mw). The release medium was $4 \mathrm{~mL}$ of $\mathrm{pH} 7.4$ phosphate buffer (PBS) containing EDTA-2Na $(2.0 \mathrm{~g} / \mathrm{L})$. The dialysis process was performed in a $10 \mathrm{~mL}$ EP tube, which was kept in an air bath at $100 \mathrm{r} / \mathrm{min}$ and $37^{\circ} \mathrm{C}$. At programmed times, all of the dialysis medium was removed and another $4 \mathrm{~mL}$ of fresh release medium were added to maintain the sink condition. The drug concentration in the outer medium was determined by the UV detector, and the in vitro release curve of the cumulative drug was determined at different times. The drug that was released from the NLC was plotted using six model-dependent approaches (zero order, first order, Higuchi's kinetics, HixsonCrowell, Weibull and Korsmeyer-Peppas) to evaluate the drug release mechanism.

\section{In vitro stability in plasma and a liver slurry}

To investigate the stability of breviscapine in a physiological environment, an in vitro stability study was conducted in fresh plasma and in the liver slurry of male Wistar rats. The plasma was diluted with saline to $67 \%$ at $37^{\circ} \mathrm{C}$, and the uniform liver slurry was homogenized with saline under an ice-bath condition. The Bre-NLC or the Bre-solution (200 $\mu \mathrm{L}$ each) was added to the plasma or the liver slurry $\left(3800 \mu \mathrm{L}\right.$ each) at $37^{\circ} \mathrm{C}$. At predetermined times, $100 \mu \mathrm{L}$ of the samples were taken from the incubation mixture and were processed immediately. All of the experiments were conducted in triplicate.

The processing method of the sample was adapted from the literature ${ }^{[28]}$. A $2 \mathrm{~mol} / \mathrm{L}$ phosphoric acid solution, $20 \mu \mathrm{L}$, was added to the plasma or liver slurry samples $(100 \mu \mathrm{L})$. The sample was vortexed at room temperature for $2 \mathrm{~min}$. Then, $500 \mu \mathrm{L}$ of methanol was added and vortexed for another 5 min. After centrifugation at $18000 \times g$ for $10 \mathrm{~min}, 20 \mu \mathrm{L}$ of the supernatant were directly injected into the HPLC column for analysis.

\section{Pharmacokinetic study}

All of the animal procedures were approved by the Institute Laboratory Animal guidelines of Sichuan University in China. Prior to the experiment, the animals were fasted but had free access to water for $12 \mathrm{~h}$. The rats were randomly divided into 2 groups with 5 animals per group and were given either the commercial drug solution or the NLC via their tail vein with a dose equivalent to $10 \mathrm{mg} / \mathrm{kg}$ breviscapine ${ }^{[7]}$. Approximately $0.3 \mathrm{~mL}$ blood samples were collected at predetermined time intervals and were centrifuged at $2300 \times g$ for $10 \mathrm{~min}$ to obtain $100 \mu \mathrm{L}$ samples. These samples were processed as described above and analyzed by HPLC. 


\section{Results}

\section{Preparation of NLC}

This study is the first research that uses an ionic complex to prepare breviscapine NLC. In this study, we developed a novel, economical and reproducible method for preparing NLC (Figure 2). It is not easy for breviscapine to form an ionic complex because of its low solubility in dichloromethane. To overcome this problem, a breviscapine-phospholipid complex was first prepared to change the solubility of the drug. The phospholipid complex then formed an ionic complex with octadecylamine. Finally, the preparation could be conducted in dichloromethane at room temperature to form NLC ${ }^{[19,29,30]}$. Ultimately, high-pressure homogenization was used to prepare the NLC. The highlight of this method is that it is applicable to industrial scale-up production.

\section{Optimal formulation}

Orthogonal design, which is a classical approach for formulation screening, was used for optimization. This method reduced the number of experiments and acquired the most valuable information. In the present study, three levels were defined for each factor, and four factors were involved in the experiment. A total of nine experiments were conducted in this design. $R$, which is called the variance, indicates the magnitude of the impact of the factors on the results. As shown in Table 2, the amount of phospholipid complex played a dominant role in the prescription. $K$ represents the average value for the same factor level. A smaller value indicates that the factor is better at this level. To obtain a better average size and a smaller PDI, which indicates uniform nanoparticles, the optimum design contained $10 \mathrm{mg}$ of breviscapine, $120 \mathrm{mg}$ of E80,
$40 \mathrm{mg}$ of MCT and $4 \mathrm{mg}$ of ODA.

\section{Encapsulation efficiency}

In our experiment, the ultrafiltration method was used to study the entrapment efficiency. The interception molecular weight of the ultrafiltration membrane is approximately 300 $\mathrm{kDa}$, and the molecular weight of breviscapine is approximately $462 \mathrm{Da}$. The free drug could pass through the ultrafiltration membrane, while breviscapine wrapped in the NLC could not. The encapsulation efficiency of the NLC was $88.95 \% \pm 0.33 \%(n=3)$.

\section{The surface nature of NLC research}

The zeta potential, mean particle size and PDI of the nanoparticles that were based on the optimized prescription were 19.8 $\mathrm{mV}, 172 \mathrm{~nm}$ and 0.157, respectively, according to the Malvern particle size analyzer. The nanoparticles were uniform with a narrow PDI distribution of less than 0.2. The Bre-NLCs were almost spherical, as shown in the transmission electron microscopy (TEM) image in Figure 3. According to the TEM image, the mean particle size of the Bre-NLC was uniform and approximately $100 \mathrm{~nm}$.

\section{In vitro release kinetics}

Breviscapine release was determined by the in vitro dialysis method. Breviscapine release from the nanoparticles was much slower than that in the commercial solution, as shown in Figure 4. The Bre-NLC exhibited a sustained release over $8 \mathrm{~h}$; subsequently, the release system reached equilibrium. After $48 \mathrm{~h}$, the cumulative drug release was $50.53 \% \pm 0.25 \%$ and $88.56 \% \pm 1.82 \%$ for the nanoparticles and the solution, respec-

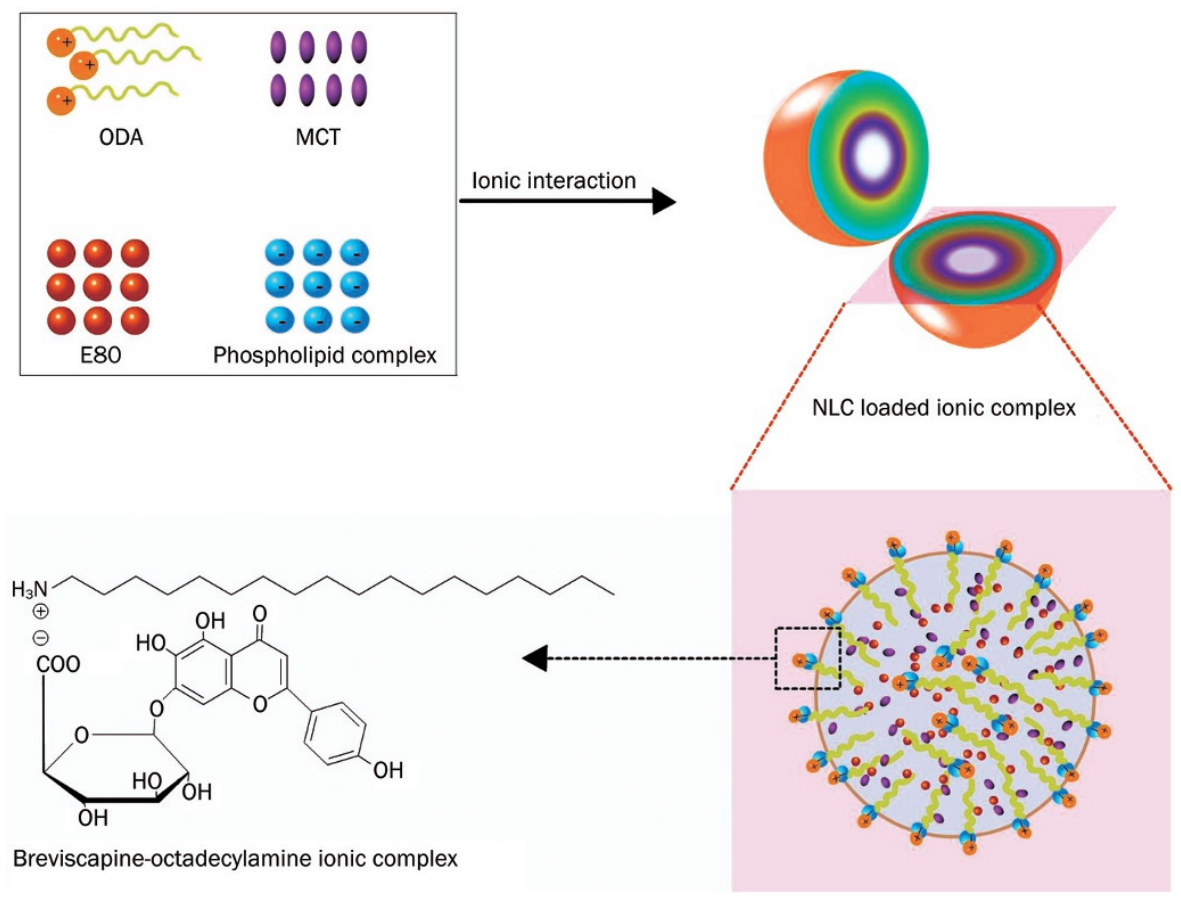

Figure 2. Bre-NLC formation based on ionic complex. 
Table 2. Orthogonal test L9 $\left(3^{4}\right)$ design and result.

\begin{tabular}{|c|c|c|c|c|c|c|}
\hline Run & Bre (mg) & E80 (mg) & MCT (mg) & ODA (mg) & PDI & Size $(n m)$ \\
\hline 1 & 8 & 100 & 20 & 4 & 0.236 & 0.236 \\
\hline 2 & 8 & 120 & 40 & 6 & 0.206 & 0.206 \\
\hline 3 & 8 & 140 & 60 & 8 & 0.232 & 0.232 \\
\hline 4 & 10 & 100 & 40 & 8 & 0.268 & 0.268 \\
\hline 5 & 10 & 120 & 60 & 4 & 0.253 & 0.253 \\
\hline 8 & 12 & 120 & 20 & 8 & 0.226 & 0.226 \\
\hline 9 & 12 & 140 & 40 & 4 & 0.192 & 0.192 \\
\hline $\mathrm{K} 1_{\mathrm{PDI}}$ & 0.225 & 0.242 & 0.243 & 0.227 & & \\
\hline $\mathrm{K} 2_{\mathrm{PDI}}$ & 0.262 & 0.228 & 0.222 & 0.231 & & \\
\hline$K 3_{P D I}$ & 0.213 & 0.230 & 0.230 & 0.242 & & \\
\hline $\mathrm{R}_{\text {size }}$ & 22.600 & 21.400 & 8.867 & 5.766 & & \\
\hline
\end{tabular}

Bre, breviscapine; E80, egg yolk lecithin 80; MCT, medium-chain fatty acid esters; ODA, octadecylamine; PDI, polydispersity index.

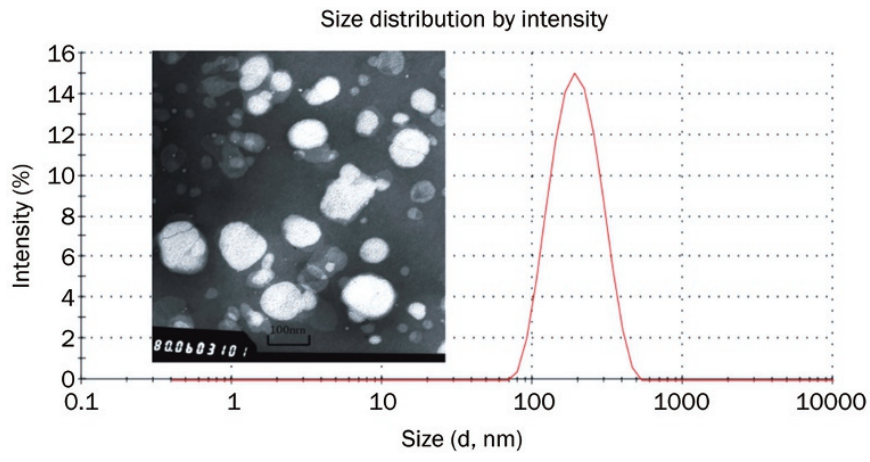

Figure 3. Transmission electron microscopy $(\mathrm{TEM})$ photograph $\left(\times 8 \times 10^{4}\right)$, particle size and PDI of Bre-NLC.

tively. A substantial decrease in the cumulative release of breviscapine was found for the nanoparticles in comparison with the commercial drug solution in PBS (pH 7.4). As shown in Table 3, of the release models, the Hixson-Crowell cube root law $\left(R^{2}=0.9795\right)$ best fit the description of the drug release mechanism. Therefore, the mechanism of breviscapine release from the NLC was an erosion process.

\section{In vitro stability evaluation}

The stability profiles of the Bre-NLC in plasma and in a liver slurry were researched. In comparison, the corresponding concentration-time profiles of the Bre-solution were studied. As shown in Figure 5, both the breviscapine solution and the NLC were relatively stable for eight hours, and there was no significant difference between them in the plasma. However, the breviscapine in the solution dramatically decreased to approximately $11 \%$ within $5 \mathrm{~min}$ in the liver slurry and almost completely degraded within $15 \mathrm{~min}$. In contrast, the brevis-

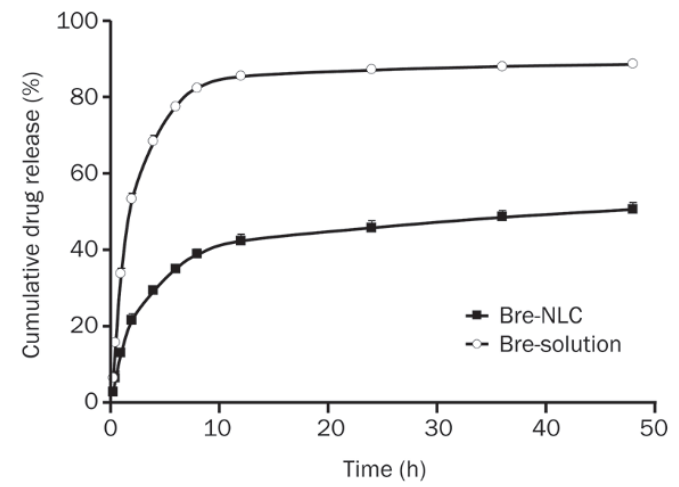

Figure 4. The in vitro drug release of Bre-NLC and commercial drug solution in PBS ( $\mathrm{pH} 7.4$ ) containing $2.0 \mathrm{~g} / \mathrm{L}$ EDTA-2Na $(n=3)$.

capine in the NLC was relatively stable with a detectable concentration at the 8-h time point.

\section{Pharmacokinetic profiles}

Figure 6 shows the breviscapine concentration in the blood at different times. The pharmacokinetics parameters were calculated by the DAS program.

After injection, the blood level of breviscapine was much higher in the case of the nanoparticles than that in the case of the solution. Breviscapine was still detectable in the plasma after $12 \mathrm{~h}$ for the Bre-NLC group, while the drug could not be detected after $1.5 \mathrm{~h}$ for the Bre-solution group. The $\mathrm{MRT}_{0-\mathrm{t}}$ of the Bre-NLC and commercial drug solutions were 186.927 \pm $16.161 \mathrm{~min}$ and $8.297 \pm 0.632 \mathrm{~min}$, respectively, and the $\mathrm{CL}_{0-\mathrm{t}}$ were $0.579 \pm 0.102 \mathrm{~L} \cdot \mathrm{min}^{-1} \cdot \mathrm{kg}^{-1}$ and $19.357 \pm 3.023 \mathrm{~L} \cdot \mathrm{min}^{-1} \cdot \mathrm{kg}^{-1}$. The half-life of the Bre-NLC and the Bre-solution were $155.782 \pm 24.777 \mathrm{~min}$ and $13.198 \pm 4.673 \mathrm{~min}$, respectively. The 
Table 3. Correlation coefficients $\left(R^{2}\right)$ of different models to describe breviscapine released from NLC .

\begin{tabular}{llll}
\hline \multicolumn{1}{c}{ Model } & \multicolumn{1}{c}{ Formula } & \multicolumn{1}{c}{ Equation } \\
\hline Zero-order & $\mathrm{M}_{t} / \mathrm{M}_{\infty}=\mathrm{kt}$ & $\mathrm{M}_{t} / \mathrm{M}_{\infty}=74.248 t-9.6212$ & 0.6177 \\
First-order & $\mathrm{M}_{t} / \mathrm{M}_{\infty}=1-\mathrm{e}^{-\mathrm{kt}}$ & $\ln \left(\mathrm{M}_{t} / \mathrm{M}_{\infty}\right)=10.409 t+28.50$ & 0.3785 \\
Higuchi & $\mathrm{M}_{t} / \mathrm{M}_{\infty}=k t^{1 / 2}$ & $\mathrm{M}_{t} / \mathrm{M}_{\infty}=11.555 t^{1 / 2}-5764$ & 0.8277 \\
Hixson-Crowell & $\mathrm{M}_{t} / \mathrm{M}_{\infty}=\mathrm{k}_{1} t+\mathrm{k}_{2} t^{2}+\mathrm{k}_{3} t^{3}$ & $\mathrm{M}_{t} / \mathrm{M}_{\infty}=1799.3 t^{3}-1065.5 t^{2}+180.01 t-5.9398$ & 0.9795 \\
Weibull & $\mathrm{M}_{t} / \mathrm{M}_{\infty}=1-\mathrm{e}^{-(t-\alpha) / \beta}$ & $\ln \ln 1 /\left(1-\mathrm{M}_{t} / \mathrm{M}_{\infty}\right)=1.2257 \ln (t-\alpha)+3.3774$ & 0.8218 \\
Korsmeyer-Peppas & $\mathrm{M}_{t} / \mathrm{M}_{\infty}=\mathrm{kt}$ & $\log \mathrm{M}_{t} / \mathrm{M}_{\infty}=1.6988 \log t+1.7597$ & 0.8726 \\
\hline
\end{tabular}

In the equation $\mathrm{M}_{t} / \mathrm{M}_{\infty}$ is fraction of drug released in time $t$, and $\mathrm{k}$ is the rate constant. In addition, $R^{2}$ is used to evaluate the model consistent with the release curve.
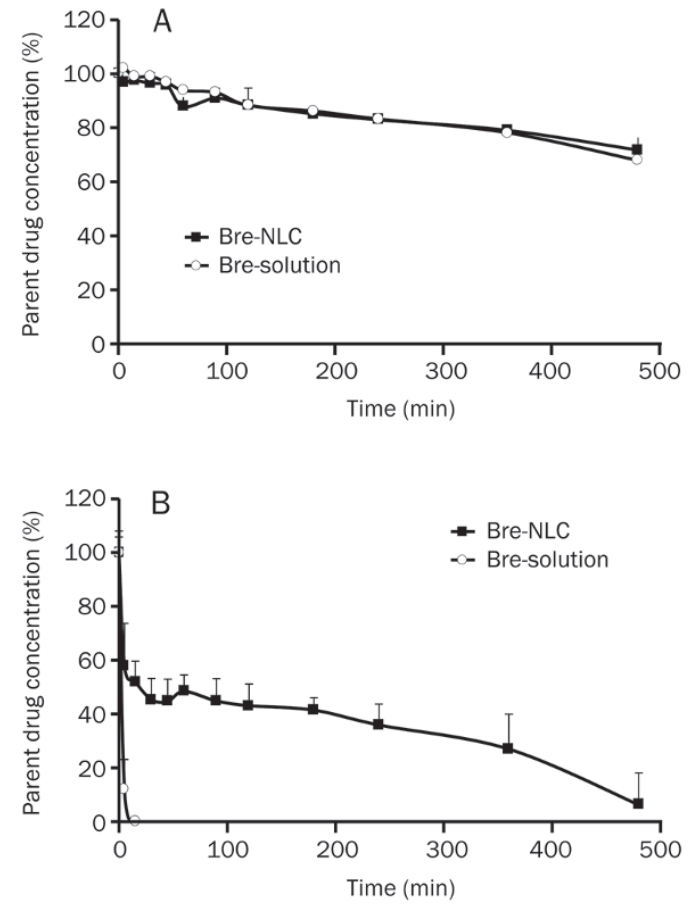

Figure 5. Stability profiles of Bre-NLC and Bre-solution obtained in rat plasma (A) and liver slurry (B) $(n=3)$.

slow elimination rate resulted in a prolonged drug residence in the blood. There was approximately a 32-fold increase in the $\mathrm{AUC}_{0-\mathrm{t}}$ of the Bre-NLC group $\left(17070.96 \pm 2717.154 \mu \mathrm{g} \cdot \mathrm{L}^{-1} \cdot \mathrm{min}\right)$ when compared with that of the Bre-solution (512.489 \pm 68.496 $\left.\mu \mathrm{g} \cdot \mathrm{L}^{-1} \cdot \mathrm{min}\right)$. It was clearly revealed that the pharmacokinetics of breviscapine significantly improved when it was delivered through the nanoparticles instead of through the commercial solution.

\section{Discussion}

In recent years, significant efforts have been devoted to developing delivery systems that increase the blood drug concentration and prolong the circulation time of breviscapine. Generally, nanostructured lipid carriers (NLC) can be used as a drug delivery system to improve the pharmacokinetic profiles, although NLC-encapsulated breviscapine has yet to

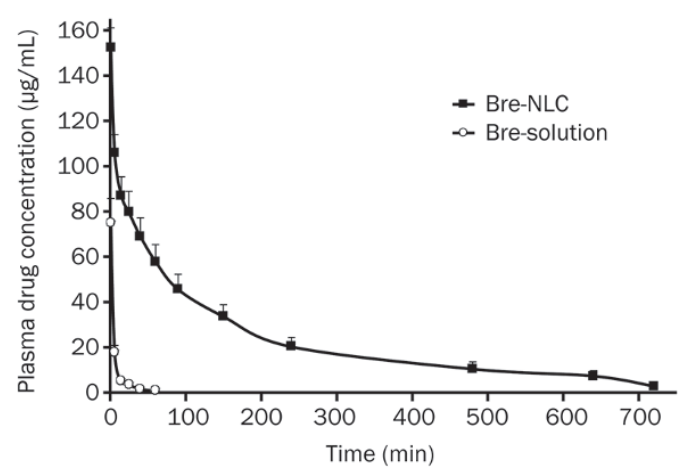

Figure 6. Mean plasma concentration-time curves of Bre-NLC and commercial drug solution in rats after intravenous administration at equivalent breviscapine dose of $10 \mathrm{mg} / \mathrm{kg}$ (mean $\pm S D, n=5)$.

be reported. Breviscapine has a low solubility, either in water or in a lipid, which was a challenge that needed to be overcome in the preparation of NLC. An ionic complex formation between octadecylamine and breviscapine was designed to solve this problem. Using a facile orthogonal design, the optimum prescription was easily obtained.

It has been reported that NLC have good drug-loading capability ${ }^{[31]}$. The lipophilicity of the drug is closely related to its incorporation ${ }^{[32,33]}$. Thus, a high breviscapine encapsulation efficiency was successfully achieved in our study due to the formation of an ionic complex. Octadecylamine has a long fatty chain and an ammonium group. The ammonium group combined with the carboxyl group of breviscapine, while the nonpolar long chain of octadecylamine produced good lipophilicity. Octadecylamine acted as a bridge to link breviscapine with a fat-soluble substance during the formation of the nanoparticles ${ }^{[34]}$. It was also found that it is difficult to incorporate breviscapine into nanoparticles without octadecylamine. When we tried to prepare nanoparticles without adding octadecylamine, breviscapine easily leaked out, even in the form of a phospholipid complex. This result indicated that the ionic complex played an irreplaceable role in breviscapine encapsulation. The resultant nanoparticles had a positive zeta potential $(19.8 \mathrm{mV})$, which could be attributed to the octadecylamine with an added ammonium group ${ }^{[35]}$. It has been demonstrated that nanoparticles with a positive charge are 
good for biological applications ${ }^{[36]}$. Additionally, the Malvern Nano-ZS90 laser particle size results showed that the BreNLC had a narrow particle dispersion of $\sim 170 \mathrm{~nm}$. However, the TEM image indicated that the nanoparticles were slightly smaller in size, which might be due to the easier adsorption of small particles onto the carbon-coated polyester fibers.

An in vitro release study, which is a routine quality control test, is an important step in the development of a new preparation. Breviscapine release from the Bre-NLC occurred in a very slow manner with a cumulative release of $\sim 50 \%$ over $48 \mathrm{~h}$. The Hixson-Crowel model matched the release style $\left(R^{2}=0.9795\right)$ well. The Hixson-Crowell cube root law indicated that the drug was released from the system by progressive dissolution, in which there is a change in the surface area and diameter of the particles ${ }^{[37-39]}$. It was proven that the mechanism of breviscapine release from the NLC was controlled by an erosion process. Without being released from the nanoparticles, it is hard for encapsulated breviscapine to go through the dialysis bag. This sustained release profile also indicated that the drug was encapsulated in the lipid core and that the nanostructure influenced the in vitro release ${ }^{[40]}$.

To speculate on the stability of the Bre-NLC in vivo, the stability profiles of the Bre-NLC and the Bre-solution in plasma and in a liver slurry were investigated, which showed that the stability of breviscapine greatly increased after encapsulation in the NLC when incubated in the liver slurry. $\beta$-Glucuronidase, which is highly expressed in the liver, is the main enzyme that is responsible for hydrolyzing a glucuronide conjugate ${ }^{[41]}$. Breviscapine could be hydrolyzed by $\beta$-glucuronidase into aglycone ${ }^{[42,43]}$, which would result in a fast decrease in the Bre-solution in the liver. Conversely, breviscapine was encapsulated in the NLC at a sustained rate. Therefore, the NLC could successfully protect breviscapine from enzyme-mediated degradation, resulting in an enhancement of the stability of breviscapine in vivo.

Pharmacokinetic studies were also performed to further understand the characteristics of the Bre-NLC in vivo. The results suggested that the Bre-NLC could remain in circulation in vivo longer than the Bre-solution, resulting in a higher $\mathrm{AUC}_{0-\mathrm{t}}$ (32-fold). The following reasons may explain the differences between the two groups. First, it has been proven that colloidal drug carriers that are based on an ionic complex can improve the AUC in vivo ${ }^{[44,45]}$. It would be more difficult for a drug to be released in the form of an ionic complex encapsulated in nanoparticles; therefore, the long-circulating effect was enhanced. Second, NLC are stealthy due to their special material and surface properties ${ }^{[12,46]}$, so they can escape from the phagocytosis of the reticuloendothelial system. Furthermore, the tight structure of the NLC, which is composed of both solid and liquid lipids, can protect a drug from enzyme degradation before release. Conversely, the Bre-solution rapidly accumulated in the liver after injection and was quickly metabolized, leading to the low AUC of the Bre-solution ${ }^{[7]}$. Due to the slow release and metabolism rate, the pharmacokinetic profile of the Bre-NLC in vivo was improved.

In summary, octadecylamine facilitated the encapsulation of breviscapine in nanoparticles by forming an ionic complex. The NLC prolonged breviscapine release into the blood and protected breviscapine from enzyme metabolism. The pharmacokinetic parameters showed that the NLC that were based on an ionic complex extended the drug residence time in the circulatory system.

\section{Conclusion}

We successfully prepared a breviscapine ionic complex and then incorporated it into NLC using the thin film homogenization method. Characterization proved that the nanoparticles had a spherical shape, a narrow distribution of approximately $170 \mathrm{~nm}$ and a high entrapment efficiency. The in vitro release study showed the sustained-release effect of the NLC. The stability experiment indicated that the NLC could protect breviscapine from liver enzyme degradation. There were significant increases in the AUC and half time of the Bre-NLC compared with those of the commercially available Bre-solution. The increased blood drug concentration and the prolonged circulation time might reduce the administration frequency, thus increasing patient compliance. Therefore, the NLC has great potential for use as a novel sustained release system for breviscapine.

\section{Acknowledgements}

This work was supported by grants from the National Basic Research Program of China (973 program, № 2013CB932504), the National S\&T Major Project of China (Grant No 2012ZX09304004) and the National Natural Science Foundation of China (№ 81273443 ).

\section{Author contribution}

Mei LI performed research and wrote the paper; Tao GONG and Zhi-rong ZHANG designed research; Yong ZHENG contributed new reagents or analytic tools; Jing ZHOU analyzed data; and Feng-ying SHAN wrote the paper.

\section{References}

1 Zhang J, Li X, Zhang W. The advance in studies on chemical component and pharmacology of Erigeron breviscapus. J Pharm Pract 2002; 20: 103-7.

2 He W, Zeng FD. Effect of breviscapine on Ischemic cerebrovascular disease and its clinical investigation. Chin J Clin Pharmacol 2002; 6: 458-61.

3 Cui J, Wu S. The advance on the research of breviscapine. Nat Prod Res Dev 2003; 15: 255-8.

4 Liu X, Yang L, Wang X, Xu H, Zhao H. Determination of scutellarin in rat plasma by HPLC and its pharmacokinetics. Chin J Pharm 2007; 38: 525.

5 Zhong H, Deng Y, Wang X, Yang B. Multivesicular liposome formulation for the sustained delivery of breviscapine. Int J Pharm 2005; 301: 15-24.

6 Lv W, Guo J, Li J, Wang X, Li J, Ping Q. Preparation and pharmacokinetics in rabbits of breviscapine unilamellar vesicles. Drug Dev Ind Pharm 2006; 32: 309-14.

7 Liu M, Li H, Luo G, Liu Q, Wang Y. Pharmacokinetics and biodistribution of surface modification polymeric nanoparticles. Arch Pharm Res 2008; 31: 547-54. 
8 Wissing S, Kayser O, Müller R. Solid lipid nanoparticles for parenteral drug delivery. Adv Drug Deliver Rev 2004; 56: 1257-72.

9 Müller RH, Mäder K, Gohla S. Solid lipid nanoparticles (SLN) for controlled drug delivery - a review of the state of the art. Eur J Pharm Biopharm 2000; 50: 161-77.

10 Cevc G, Vierl U. Nanotechnology and the transdermal route: A state of the art review and critical appraisal. J Control Release 2010; 141: 277-99.

11 Angst MS, Drover DR. Pharmacology of drugs formulated with DepoFoam: a sustained release drug delivery system for parenteral administration using multivesicular liposome technology. Clin Pharmacokinet 2006; 45: 1153-76.

12 Li X, Nie S, Kong J, Li N, Ju C, Pan W. A controlled-release ocular delivery system for ibuprofen $b$ ased on nanostructured lipid carriers. Int J Pharm 2008; 363: 177-82.

13 Muller RH, Keck CM. Challenges and solutions for the delivery of biotech drugs - a review of drug nanocrystal technology and lipid nanoparticles. J Biotechnol 2004; 113: 151-70.

14 Müller R, Olbrich C. Drug-delivery vehicle for the controlled administration of an active agent, produced from lipid matrix-medicament conjugates. PCT Int Appl WO 2000; 2000067800.

15 Joshi MD, Müller RH. Lipid nanoparticles for parenteral delivery of actives. Eur J Pharm Biopharm 2009; 71: 161-72.

16 Smith RM, Alberty RA. The apparent stability constants of Ionic complexes of various adenosine phosphates with divalent cations1, 2 . J Am Chem Soc 1956; 78: 2376-80.

17 Bieske E, Maier J. Spectroscopic studies of ionic complexes and clusters. Chem Rev 1993; 93: 2603-21.

18 Polfer NC, Oomens J. Vibrational spectroscopy of bare and solvated ionic complexes of biological relevance. Mass Spectrom Rev 2009; 28: 468-94.

19 Tominaga Y, Kubo T, Kaya K, Hosoya K. Effective recognition on the surface of a polymer prepared by molecular imprinting using ionic complex. Macromolecules 2009; 42: 2911-15.

20 Balapanuru J, Yang JX, Xiao S, Bao Q, Jahan M, Polavarapu L, et al. A graphene oxide-organic dye ionic complex with DNA - Sensing and optical-limiting properties. Angewandte Chemie 2010; 122: 6699-703.

21 Kim YJ, Chae SY, Jin CH, Sivasubramanian M, Son S, Choi KY, et al. Ionic complex systems based on hyaluronic acid and PEGylated TNF-related apoptosis-inducing ligand for treatment of rheumatoid arthritis. Biomaterials 2010; 31: 9057-64.

22 Pino T, Boudin N, Bréchignac P. Electronic absorption spectrum of cold naphthalene cation in the gas phase by photodissociation of its van der Waals complexes. J Chem Phys 1999; 111: 7337.

23 Lv W, Guo J, Li J, Huang L, Ping Q. Distribution of liposomal breviscapine in brain following intravenous injection in rats. Int J Pharm 2005; 306: 99-106.

24 Pardeike J, Hommoss A, Müller RH. Lipid nanoparticles (SLN, NLC) in cosmetic and pharmaceutical dermal products. Int J Pharm 2009; 366: $170-84$.

25 Fang KT, Wang Y, Bentler PM. Some applications of number-theoretic methods in statistics. Stat Sci 1994; 9: 416-28.

26 Hedayat AS, Sloane NJA, Stufken J. Orthogonal arrays: theory and applications. Springer; 1999.

27 Peng Q, Zhang ZR, Sun X, Zuo J, Zhao D, Gong T. Mechanisms of phospholipid complex loaded nanoparticles enhancing the oral bioavailability. Mol Pharm 2010; 7: 565-75.

28 Xiong $\mathrm{F}$, Wang $\mathrm{H}$, Cheng J, Zhu J. Determination of scutellarin in mouse plasma and different tissues by high-performance liquid chromatography. J Chromatogr B 2006; 835: 114-8.

29 Guo Y, Liu X, Sun X, Zhang Q, Gong T, Zhang Z. Mannosylated lipid nano-emulsions loaded with lycorine-oleic acid ionic complex for tumor cell-specific delivery. Theranostics 2012; 2: 1104-14.

30 Choi SH, Park TG. Hydrophobic ion pair formation between leuprolide and sodium oleate for sustained release from biodegradable polymeric microspheres. Int J Pharm 2000; 203: 193-202.

31 Galeano NF, Milne R, Marcel YL, Walsh MT, Levy E, Ngu'yen TD, et al. Apoprotein $B$ structure and receptor recognition of triglyceriderich low density lipoprotein (LDL) is modified in small LDL but not in triglyceride-rich LDL of normal size. J Biol Chem 1994; 269: 511-9.

$32 \mathrm{Hu} F Q$, Jiang SP, Du YZ, Yuan H, Ye YQ, Zeng S. Preparation and characterization of stearic acid nanostructured lipid carriers by solvent diffusion method in an aqueous system. Colloid Surface B 2005; 45: 167-73.

33 Zhang X, Pan W, Gan L, Zhu C, Gan Y, Nie S. Preparation of a dispersible PEGylate nanostructured lipid carriers (NLC) loaded with 10-hydroxycamptothecin by spray-drying. Chem Pharm Bull 2008; 56 : 1645-50.

34 Lee YL, Yang YC, Shen YJ. Monolayer characteristics of mixed octadecylamine and stearic acid at the air/water interface. J Phys Chem B 2005; 109: 4662-7.

35 Ralston A, Hoerr CW, Hoffman EJ. Studies on high molecular weight aliphatic amines and their salts. VII. The Systems Octylamine-, Dodecylamine-, and octadecylamine-water. J Am Chem Soc 1942; 64: 1516-23.

36 Yu B, Zhang Y, Zheng W, Fan C, Chen T. Positive surface charge enhances selective cellular uptake and anticancer efficacy of selenium nanoparticles. Inorg Chem 2012; 51: 8956-63.

37 Ritger PL, Peppas NA. A simple equation for description of solute release I. Fickian and non-Fickian release from non-swellable devices in the form of slabs, spheres, cylinders or discs. J Control Release 1987; 5: 23-36.

38 Bisrat M, Nyström C. Physicochemical aspects of drug release. VIII. The relation between particle size and surface specific dissolution rate in agitated suspensions. Int J Pharm 1988; 47: 223-31.

39 Hixon AW, Crowel JH. Dependence of reaction velocity upon surface and agitation. I-Theoretical considerations. Ind Eng Chem 1931; 23 : 923-31.

40 Joshi M, Pathak S, Sharma S, Patravale V. Design and in vivo pharmacodynamic evaluation of nanostructured lipid carriers for parenteral delivery of artemether: Nanoject. Int J Pharm 2008; 364: 119-26.

41 O'Leary KA, Day AJ, Needs PW, Mellon FA, O'Brien NM, Williamson G. Metabolism of quercetin-7- and quercetin-3-glucuronides by an in vitro hepatic model: the role of human $\beta$-glucuronidase, sulfotransferase, catechol-O-methyltransferase and multi-resistant protein 2 (MRP2) in flavonoid metabolism. Biochem Pharmacol 2003; 65: 479-91.

42 Zhang JL, Che QM, Li SZ, Zhou TH. Study on metabolism of scutellarin in rats by HPLC-MS and HPLC-NMR. J Asian Nat ProdP Res 2003; 5: 249-56.

43 Chen X, Cui L, Duan X, Ma B, Zhong D. Pharmacokinetics and metabolism of the flavonoid scutellarin in humans after a single oral administration. Drug Metab Dispos 2006; 34: 1345-52.

44 Nguyen MK, Huynh CT, Gao GH, Kim JH, Chae SY, Lee KC, et al. Biodegradable oligo (amidoamine/ $\beta$-amino ester) hydrogels for controlled insulin delivery. Soft Matter 2011; 7: 2994-3001.

45 Zhang X, Sun X, Li J, Zhang X, Gong T, Zhang Z. Lipid nanoemulsions loaded with doxorubicin-oleic acid ionic complex: characterization, in vitro and in vivo studies. Pharmazie 2011; 66: 496-505.

46 Zhuang CY, Li N, Wang M, Zhang XN, Pan WS, Peng JJ, et al. Preparation and characterization of vinpocetine loaded nanostructured lipid carriers (NLC) for improved oral bioavailability. Int J Pharm 2010; 394: $179-85$. 